\title{
Irradiation effects in semiconductor
}

\author{
V N BHORASKAR \\ Department of Physics, University of Pune, Pune 411 007, India
}

\begin{abstract}
Samples of crystalline silicon, porous silicon, gallium arsenide and silicon diodes were exposed to $50-80 \mathrm{MeV}$ silicon and oxygen ions in the fluence range of the order of $10^{13}$ to $10^{14} \mathrm{ions} / \mathrm{cm}^{2}$. The irradiated samples were characterized to obtain information on the relative concentration and depth distribution of the induced defects. For comparison a few silicon diodes and crystalline silicon samples were also exposed to $6 \mathrm{MeV}$ electrons. The main techniques used for the analysis of silicon samples were low angle X-ray diffraction, photoluminescence spectroscopy and lifetime of minority carriers, whereas diodes were characterized on the basis of switching parameters. It is observed that a large number of defects are produced in the surface region of each of the irradiated semiconductor sample though the energy deposited in the surface region through electronic loss is three orders of magnitude greater than that of nuclear collisions.
\end{abstract}

Keywords. Silicon; ions; defects; irradiation; diodes; lifetime; minority carriers.

\section{Introduction}

A large number of reports dealing with the effects of low energy heavy ions on semiconductor materials are available in the literature, however, relatively a few reports are available describing effects of high energy heavy ions on semiconducting materials and devices (Schultz et al 1991; Railkar et al 1993; Zhu et al 1993). The energy loss mechanism for low energy heavy ions has been well studied and the experimental results match fairly well with the theoretical predictions. In the case of high energy heavy ions, though the theories for the energy loss in a medium are well established, however, in several cases the experimental results do not agree with the theory.

A high energy ion upon entering a medium loses energy initially through the process of electronic excitation and ionization, known as electronic energy loss, and at the end of its range, loses energy mostly through the process of nuclear loss, in which the lattice atoms of the medium are displaced due to nuclear elastic collisions. The latter phenomenon of energy loss becomes prominent when the ion energy reduces to $\sim \mathrm{A} \mathrm{KeV}$, where $\mathrm{A}$ is the atomic weight of the ion. In the surface region of the sample through which an ion enters, the energy loss suffered by a heavy ion through electronic excitation is greater by three orders of magnitude than that through nuclear elastic collisions. In this way the energy deposited in the surface region through elastic collisions should have negligible contribution in defect formation. As such in the sample exposed to high energy heavy ions, the atoms in the surface region are not expected to get displaced from their lattice positions. The other processes through which defects may be induced in the surface region of a sample exposed to heavy ions are Coulomb explosion (Hansen et al 1982) and heat spike (Zhu et al 1993). However, theories for these processes are not well established. In order to find out whether an appreciable number of defects are induced in the surface region of a sample exposed to high energy heavy ions, we have carried out a systematic study by exposing crystalline silicon, porous silicon, gallium arsenide and silicon diode samples to $50-80 \mathrm{MeV}$ energy silicon and oxygen ions. The level of induced damage in each 
irradiated sample was estimated by comparing pre-irradiation characteristics with post-irradiation characteristics.

\section{Experimental}

Phosphorous doped crystalline silicon wafers of $160 \Omega-\mathrm{cm}$ resistivity and $300 \mu \mathrm{m}$ thickness were cut to make samples of size $10 \mathrm{~mm} \times 12 \mathrm{~mm}$. In addition, silicon diode wafers, porous silicon and $\mathrm{GaAs}$ samples were also used. Using the Pelletron facility of the Nuclear Science Centre, New Delhi, samples were exposed in turn, to $50-80 \mathrm{MeV}$ energy silicon and oxygen ions with different fluences in the range, $10^{13}-10^{14}$ ions $/ \mathrm{cm}^{2}$. In an another experiment, wafers of silicon diodes were exposed from p-side to $60 \mathrm{MeV}$ oxygen ions. The fluences used were in the range $5 \times 10^{13}$ to $5 \times 10^{14}$ ions $/ \mathrm{cm}^{2}$. Similarly, four samples of porous silicon prepared at different current densities were exposed to $60 \mathrm{MeV}$ silicon ions. Using the Race-track microtron facility (Asgekar et al 1980 ) of this laboratory another set of four silicon samples and diodes were exposed to $6 \mathrm{MeV}$ electrons of four different fluences in the range $10^{14}-10^{15} \mathrm{e} / \mathrm{cm}^{2}$. In case of oxygen and silicon ions, the ion beam was defocused to obtain uniform intensity across the sample surface, covering a rectangular area $\sim 8 \mathrm{~mm}$ in length and $5 \mathrm{~mm}$ in width. During irradiation the charge collected by the sample holder was measured by a current integrator, and from this the ion fluence was estimated. In case of electron irradiation, the electron beam was scattered and a slit arrangement was used to obtain a rectangular beam, $\sim 8 \mathrm{~mm}$ in length and $5 \mathrm{~mm}$ in width, on the sample.

Silicon diodes used for this study were made without gold diffusion. Characteristics of these diodes such as turn off time $t_{\pi}$, storage time $t_{\mathrm{s}}$, and forward voltage drop $V_{\mathrm{F}}$, were measured and later on exposed to different fluences of $6 \mathrm{MeV}$ electrons, and 50-80 MeV oxygen and silicon ions. Post irradiation characteristics of each diode were measured. Dependence of $t_{\pi}$ and forward voltage drop $V_{F}$ on the electron and ion fluences were studied.

The ion irradiated silicon samples were analyzed using techniques of grazing angle $\mathrm{X}$-ray diffraction and lifetime of minority carriers. For a few samples, the silicon ion irradiated side was chemically etched to remove a layer of thickness $\sim 3 \mu \mathrm{m}$ at a time. The etched surface was analyzed through lifetime of minority carriers. The sample was again etched and the lifetime of minority carriers was measured. The method used for measurement of lifetime of minority carriers was the same as described in our earlier publication (Padgaonkar et al 1991). The results of the X-ray diffraction are shown in figures 1 and 2; lifetime of minority carriers are shown in figure 3 and that of porous silicon are shown in figure 4 . The irradiated GaAs samples were analyzed with thermally stimulated exoelectron emission technique (Railkar et al 1992).

\section{Results and discussion}

Results shown in figures 1 and 2 indicate that the intensities of X-ray diffraction peaks at $28.8^{\circ}(2 \theta)$ recorded for $1^{\circ}$ and $10^{\circ}$ incident angles have reduced for both oxygen and silicon ion irradiated silicon samples as compared to the unirradiated silicon sample.

The decrease in the peak intensity reveals that structural damage has been induced in the surface region of the sample. However, for heavy ions, the magnitude of the energy loss through the process of electronic excitation and ionization is much more as 


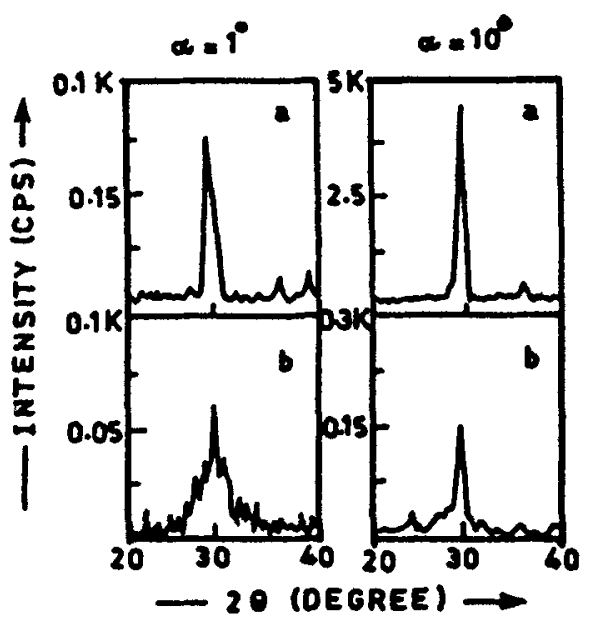

Figure 1. X-ray diffraction spectrum of (a) unirradiated silicon and (b) $80 \mathrm{MeV}$ oxygen ion irradiated $\left(\phi=5 \times 10^{13}\right.$ ions $\left./ \mathrm{cm}^{2}\right)$ crystalline silicon measured for X-ray incident angles $(\alpha)$ of $1^{\circ}$ and $10^{\circ}$.

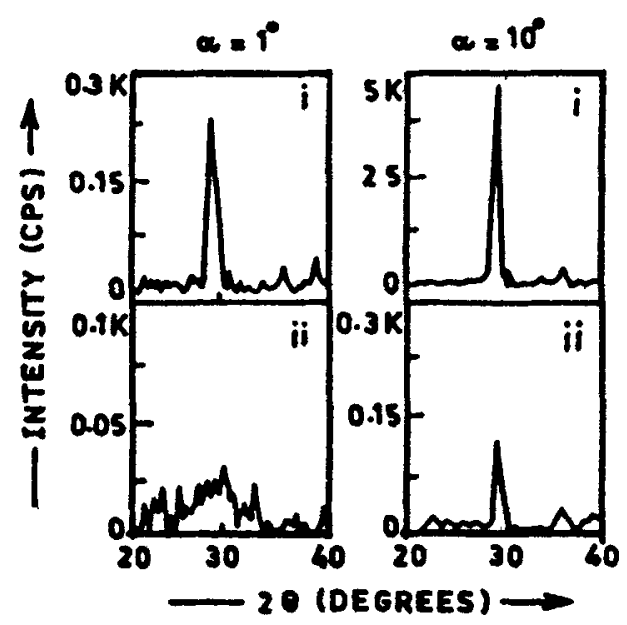

Figure 2. X-ray diffraction spectrum of (i) unirradiated and (ii) $80 \mathrm{MeV}$ silicon ion irradiated $\left(\phi=5 \times 10^{13}\right.$ ions $\left./ \mathrm{cm}^{2}\right)$ crystalline silicon measured for $X$-ray incident angles $(\alpha)$ of $1^{\circ}$ and $10^{\circ}$.

compared to that through the nuclear collisions. It is therefore surprising that such a large number of lattice atoms have been displaced in the surface region of the heavy ion irradiated silicon. Similar conclusion can be drawn from the characteristics of the silicon diodes irradiated with oxygen and silicon ions. The junction of diode is situated at a distance of $\sim 50 \mu \mathrm{m}$ from the surface of p-region, whereas the ranges of $80 \mathrm{MeV}$ oxygen ions and silicon ions in silicon are $70.52 \mu \mathrm{m}$ and $27.56 \mu \mathrm{m}$ respectively. It is, therefore, expected that a large number of lattice atoms are displaced at the end of the ion trajectory and a small number of lattice atoms are displaced in the surface region of p-side. From the results, it is observed that the forward voltage drop of the diodes irradiated with heavy ions had increased considerably, whereas the decrease in $t_{\pi}$ is not in accordance with the increase in the forward voltage drop. Had most of the atoms 


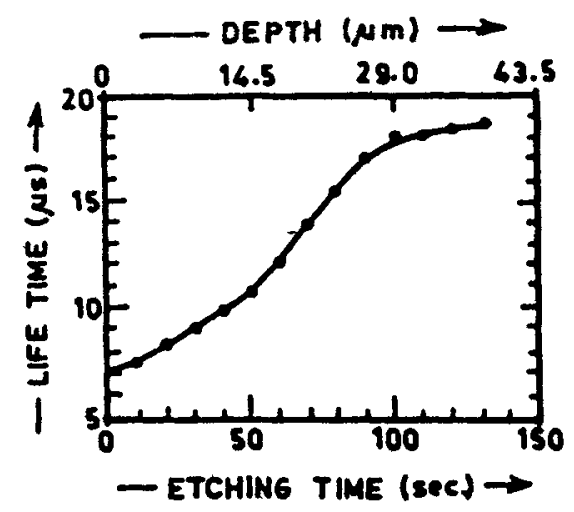

Figure 3. For an silicon ion irradiated crystalline silicon, variations in the lifetime of minority carriers with etching time. The upper scale indicates the thickness of silicon etched out.

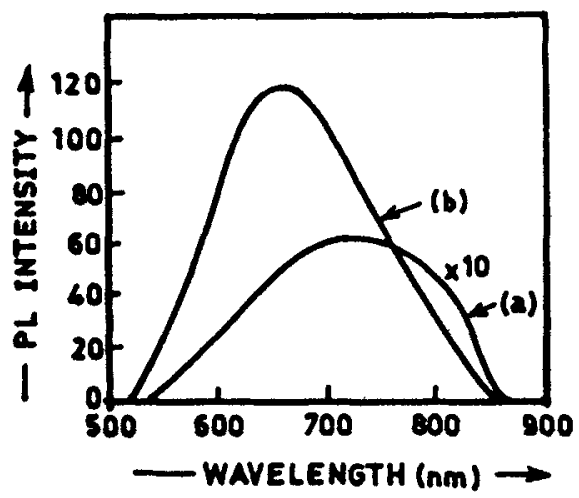

Figure 4. Photoluminescence spectra of (a) virgin porous silicon and (b) silicon ion irradiated porous silicon sample.

been displaced only at the end of the trajectory, the increase in the voltage drop would have been much smaller in magnitude. For the diodes irradiated with $6 \mathrm{MeV}$ electrons, the increase in the voltage drop is relatively small as compared to that of ion irradiated diodes. These results therefore, reveal that a large number of lattice atoms in the p-region of the diode had been displaced from surface to the projected range of the ions. The measured values of the lifetime of minority carriers in a silicon ion irradiated crystalline silicon sample after each stage of etching by $3 \mu \mathrm{m}$ are shown in figure 3 .

It is interesting to observe that on removal of the silicon layer, the lifetime of minority carriers increased. Furthermore, when the total etched layer became more than the range of heavy ions, the lifetime of minority carriers reached the value close to that of unirradiated silicon sample. These results indicate that though the cross section for energy loss through nuclear collisions is much smaller as compared to that through electronic loss, a large number of lattice atoms are displaced from the surface region. Photoluminescence spectra for a typical porous silicon sample before and after irradiation with $60 \mathrm{MeV}$ silicon ions are shown in figure 4. It is interesting to note that on ion irradiation, the peak intensity of the photoluminescence had increased by 
almost 20 times and also the peak wavelength shifted to $650 \mathrm{~nm}$ from $725 \mathrm{~nm}$. Since, the photoluminescence phenomenon is surface sensitive, these results confirm that high energy heavy ions induce defects in the surface region. The irradiated GaAs samples were analyzed with the technique of thermally stimulated exoemission. These results also revealed that in the surface regions a large number of lattice atoms had been displaced. On the basis of these results it appears that in addition to nuclear collisions, there should be another mechanism which is responsible for inducing damage in the surface region. It is known that a highly charged heavy ion upon entering a medium can produce a continuous volume of electronic excitation and ionization. As a result, rows of positively charged atoms can exist face-to-face for a time period equal to that of electronic excitation. These columns of positive charges can repel each other leading to ejection of the lattice ions in the direction perpendicular to the incident ion trajectory. In earlier studies (Hansen et al 1982), this phenomenon, called Coulomb explosion, had been found responsible for inducing defects in the surface region. In the present work the observed defects produced in the surface region of silicon by heavy ions may have contributions from both the processes, nuclear elastic collisions as well as Coulomb explosion.

\section{Acknowledgements}

Thanks are due to Prof. G K Mehta, the Director and other concerned scientists of NSC, New Delhi for providing the facility and help in conducting ion irradiation experiment.

\section{References}

Asgekar V B, Bhalla R K, Raye B S, Bhiday M R and Bhoraskar V N 1980 Pramana-J. Phys. 15479

Hansen P, Heitmann H and Smit P H 1982 Phys. Rev. B29 3539

Padgaonkát S, Dhole S D and Bhoraskar V N 1991 J. Phys. D24 702

Railkar T A, Bhide R S, Bhoraskar S V, Manorama V and Rao V J 1992 J. Appl. Phys. 72155

Railkar T A, Bhoraskar S V, Dhole S D and Bhoraskar V N 1993 J. Appl. Phys. 744343

Schultz J, Jagadish C, Ridgway M C, Elliman R G and Williams J C 1991 Phys. Rev. B44 9118

Zhu Y, Cai Z X, Budhani R C, Suenaga M and Welch D O 1993 Phys. Rev. B48 6436 